\title{
Inhibition of the SIRT1 signaling pathway exacerbates endoplasmic reticulum stress induced by renal ischemia/reperfusion injury in type 1 diabetic rats
}

\author{
JIANJIAN ZHANG ${ }^{*}$, LEI WANG* ${ }^{*}$, DAOJING GONG, YUANYUAN YANG, XIUHENG LIU and ZHIYUAN CHEN \\ Department of Urology, Renmin Hospital of Wuhan University, Wuhan, Hubei 430060, P.R. China
}

Received June 18, 2019; Accepted November 28, 2019

DOI: $10.3892 / \mathrm{mmr} .2019 .10893$

\begin{abstract}
The aim of the present study was to investigate whether the diabetic kidney is more susceptible to ischemia/reperfusion (I/R) injury, and identify the potential mechanisms involved. An animal model of type 1 diabetes was created by treating rats with streptozotocin (STZ). This model was then used, along with healthy controls, to investigate the effect of diabetes mellitus (DM) on renal I/R injury. After $45 \mathrm{~min}$ of ischemia and $24 \mathrm{~h}$ of reperfusion, kidney and serum samples were acquired and used to evaluate function and histopathological injury in the kidneys. Western blotting was also used to determine the expression levels of key proteins. Rats experiencing renal I/R exhibited significant characteristics of renal dysfunction, reduced levels of Sirtuin 1 (SIRT1) protein (a key signaling protein in the kidneys), increased endoplasmic reticulum stress (ERS) and pyroptosis. Furthermore, diabetic rats exhibited further reductions in the levels of SIRT1 in response to renal I/R injury and an increase in the levels of ERS. These effects were all alleviated by the administration of a SIRT1 agonist. The present analysis revealed that the SIRT1-mediated activation of ER stress and pyroptosis played a pivotal role in diabetic rats subjected to renal I/R injury. Downregulation of the SIRT1 signaling pathway were exacerbated in response to renal I/R injury-induced acute kidney injury (AKI). The present data indicated that DM enhanced ER stress and increased pyroptosis by downregulating the SIRT1 signaling pathway.
\end{abstract}

Correspondence to: Professor Xiuheng Liu or Professor Zhiyuan Chen, Department of Urology, Renmin Hospital of Wuhan University, 238 Jiefang Road, Wuhan, Hubei 430060, P.R. China

E-mail: drliuxh@hotmail.com

E-mail: chenzhiyuan163@163.com

*Contributed equally

Key words: type 1 diabetes mellitus, renal ischemia/reperfusion injury, SIRT1 signaling, endoplasmic reticulum stress, pyroptosis, resveratrol

\section{Introduction}

Renal ischemia/reperfusion ( $\mathrm{I} / \mathrm{R})$ injury is a leading cause of acute kidney injury (AKI) and is associated with severe morbidity and mortality in both developing and developed countries (1). A number of mechanisms have been reported to enhance the susceptibility of elderly patients to AKI (2). Diabetes mellitus (DM) is a metabolic disorder associated with a multitude of clinical syndromes, including atherosclerosis (3). In addition, DM is associated with a number of severe pathological risks, including the development of AKI $(3,4)$. Evidence has accumulated supporting the fact that DM aggravates renal I/R injury (5). Experimental studies have revealed that diabetic rats develop renal dysfunction faster following IR injury compared with non-diabetic rats $(6,7)$. Other research has revealed that progressive hyperglycemia can cause increased levels of reactive oxygen species (ROS) in the diabetic kidney following I/R injury (8). However, little is known concerning the specific mechanisms responsible for how diabetes can result in an increased vulnerability to $I / R$ injury.

The endoplasmic reticulum (ER) is an intracellular organelle that plays a key role in protein homeostasis, including protein folding, processing, conveyancing and degradation (9). However, the ER is also susceptible to a range of stressors, including hypoxia, $\mathrm{Ca}^{2+}$ overload, I/R and ROS (10). Stimulation of the ER by one or more of these stimuli can cause the production of numerous unfolded or misfolded proteins and the initiation of the unfolded protein response (UPR), eventually leading to the activation of ERS (11). Three UPR pathways have been described, each named after a transmembrane regulator: Inositol-requiring enzyme $1 \alpha$ (IRE1 $\alpha$ ), protein kinase R-like ER kinase (PERK) and activating transcription factor 6 (ATF6) (12). A large body of data has also demonstrated that renal $\mathrm{I} / \mathrm{R}$ injury can induce ERS and cause AKI (13-15). In addition, in animal models of diabetes, hyperglycemia can induce the glycation of proteins and thus impose an enormous burden on the ER with regards to the abnormal refolding of misfolded or unfolded proteins; this can result in ERS-induced apoptosis (16). It was therefore hypothesized that there is a relationship between ERS and I/R injury in the diabetic kidney. Pyroptosis manifests as a type of inflammatory programmed cell death induced by inflammatory caspases and exhibits morphological characteristics 
that are common to apoptosis and necrosis (17). However, unlike necrosis or apoptosis, pyroptosis results in the activation of pro-inflammatory mediators that are triggered by the release of cytokines (18). Initially, pyroptosis activates caspase- 1 and then induces the production of the inflammatory cytokine interleukin-1 $\beta$ (IL-1 $\beta$ ) (19). Notably, pyroptosis plays a key role during renal $\mathrm{I} / \mathrm{R}$ injury; the overactivation of ERS via the $\mathrm{CHOP} /$ caspase-11 signaling pathway has also been revealed to produce a significant contribution to this process (20).

Sirtuin 1 (SIRT1) is an NAD ${ }^{+}$-dependent histone deacetylase that uses deacetylating multiple factors to regulate a variety of biological processes, including cell metabolism, gene transcription, immunological response and glucose homeostasis $(21,22)$. A recent study demonstrated that SIRT1 can protect renal function by physically interacting with and deacetylating eIF2 $\alpha$ at lysine (K143) residues (23). This action inhibits the PERK-eIF2 $\alpha$-ATF4/CHOP axis, thus attenuating ERS-mediated apoptosis (23). Furthermore, it has been reported that diabetic rats exhibit reduced levels of SIRT1 signaling $(7,24)$. Notably, research has revealed that SIRT1 is downregulated by $\mathrm{I} / \mathrm{R}$ injury and that the overactivation of SIRT1 attenuates I/R-induced myocardial damage $(7,25)$. However, whether SIRT1 signaling is downregulated in diabetes-exacerbated renal I/R injury, and whether ERS is involved in this process, remains unknown.

The present study aimed to investigate the potential mechanisms mediating diabetes-aggravated renal I/R injury with specific emphasis on SIRT1 signaling and its association with ERS, and the potential role of pyroptosis.

\section{Materials and methods}

Animal models. A total of 30 adult male Sprague-Dawley (SD) rats, aged 6-8 weeks, weighing 220-250 g, were obtained from the Center of Experimental Animals in the Medical College of Wuhan University. The animals were placed in a room with a temperature of $23 \pm 3^{\circ} \mathrm{C}$ and relative humidity of $40-70 \%$, with $12 \mathrm{~h}$ day/night cycles. They were provided with water and a standard diet ad libitum. This study was approved by the Ethics Committee of Renmin Hospital of Wuhan University and all procedures complied with the Principles of Animal Care of Wuhan University (Wuhan, China) and Guide for the Care and Use of Laboratory Animals.

Materials. Streptozotocin (STZ), dimethyl sulfoxide (DMSO) and resveratrol were purchased from Sigma-Aldrich; Merck KGaA. Primary antibodies against SIRT1 (cat. no. DF6033), p-PERK (cat. no. DF7576), p-eIF2 $\alpha$ (cat. no. AF3087), ATF4 (cat. no. AF5416), CHOP (cat. no. DF6025), IL-1 $\beta$ (cat. no. DF6251), caspase-1 (cat. no. AF5418) and IL-18 (cat. no. DF6252) were purchased from Affinity Biosciences. Primary antibody against NLRP3 (cat. no. 19771-1-AP) was purchased from ProteinTech Group, Inc. Primary antibodies against PERK (cat. no. ab77654) and eIF2 $\alpha$ (cat. no. ab169528) were purchased from Abcam. A primary antibody against caspase-11 (cat. no. sc-56038) was purchased from Santa Cruz Biotechnology, Inc. A primary antibody against $\beta$-actin (cat. no. BM0627), goat anti-mouse (cat. no. BA1051) and goat anti-rabbit (cat. no. BA1054) secondary antibodies were purchased from Boster Biological Technology.

Type 1 diabetic rat model. STZ [60 mg/kg, dissolved in $0.1 \mathrm{M}$ citrate buffer ( $\mathrm{pH} 4.5)$ ] was administered to each experimental rat by intraperitoneal (i.p.) injection (26). Prior to injection, all rats were fasted for $12 \mathrm{~h}$. Normal rats received an i.p. injection with the same dose of citrate buffer. Commencing $72 \mathrm{~h}$ after the injection of STZ, blood glucose levels were measured in the tail vein of rats for 3 days in succession. Only rats exhibiting hyperglycemia (a fasting blood glucose level above $16.7 \mathrm{mmol} / \mathrm{l}$ ) were ultimately regarded as having diabetes (26).

Induction of renal ischemia/reperfusion injury. Rats were anaesthetized (i.p.) with pentobarbital ( $45 \mathrm{mg} / \mathrm{kg}$ body weight) and placed on a thermostat to maintain a body temperature of $37^{\circ} \mathrm{C}$ during surgery. In each rat, the kidneys were first exposed through a midline abdominal incision. Then, the right kidney was resected. Subsequently, the left renal pedicle was clamped for $45 \mathrm{~min}$ using non-invasive vascular forceps and then the clamp was removed for $24 \mathrm{~h}$ to allow reperfusion.

Experimental groups and protocol. The diabetic model was established in 5 weeks. Diabetic and non-diabetic rats were then randomly divided into 5 groups (6 rats per group): i) The non-diabetic sham group (NS); ii) the non-diabetic I/R group (NI/R); iii) the diabetic sham group (DS); iv) the diabetic I/R group (DI/R); v) the diabetic I/R + resveratrol group (DI/R+Res). In the sham groups, right nephrectomy was only performed. In the I/R groups, I/R injury was induced, as aforementioned. Resveratrol is a well-known agonist of SIRT1 (27); rats in the DI/R+Res group were injected with resveratrol (dissolved in DMSO and delivered with saline and $30 \%$ ethanol) at a dose of $10 \mathrm{mg} / \mathrm{kg}$ body-weight (i.p.) per day for one week; $30 \mathrm{~min}$ before surgery, these rats were administered an injection (i.p.) of the same dose (28). Rats in the other groups were injected (i.p.) with the same volume of DMSO on the same timescale.

Renal function and histological examination. Blood samples were obtained $24 \mathrm{~h}$ after reperfusion to allow the determination of serum blood urea nitrogen (BUN) and serum creatinine (Scr) using a spectrophotometer (Jiancheng Biotech). Calculation of these parameters allowed renal function to be assessed. Renal tissue samples were fixed in $4 \%$ paraformaldehyde at $4{ }^{\circ} \mathrm{C}$ for $6 \mathrm{~h}$, embedded in paraffin and sectioned at a thickness of $4 \mu \mathrm{m}$. Then, the sections were deparaffinized in dimethylbenzene at $60^{\circ} \mathrm{C}$ and hydrated in ethanol (100\% twice, $95 \%$ twice, $75 \%$ twice, distilled water). Following this the sections were stained with hematoxylin and eosin (H\&E; 4 min with hematoxylin and 4 min with eosin at room temperature) in order to assess histopathological kidney injury. Two experienced renal pathologists then used the sections to independently assess morphological changes. The severity of injury to the renal tubules was defined with 5 grades (0-4): 0 , no evident visible injury; 1 , injury $<25 \%$; 2 , injury between $25-50 \%$; 3 , injury between $50-75 \%$; and 4 , injury $>75 \%$ (29).

Western blot analysis. Samples of rat kidneys were collected and snap-frozen in liquid nitrogen. Total proteins were 
then extracted from these tissues using RIPA lysis buffer (Beyotime Institute of Biotechnology). The bicinchoninic acid (BCA) method was used to quantify protein levels prior to western blotting. In brief, protein samples (40 $\mu \mathrm{g} /$ lane) were separated on SDS-PAGE gels (5\% separating, 10\% stacking gel) and then transferred to PVDF membranes. Subsequently, PVDF membranes were blocked with 5\% non-fat milk for $2 \mathrm{~h}$ and then incubated at $4^{\circ} \mathrm{C}$ overnight with specific antibodies against SIRT1 (1:1,000), p-PERK (1:2,000), PERK $(1: 2,000)$, p-eIF2 $\alpha(1: 1,000)$, eIF2 $\alpha(1: 1,000)$, ATF4 (1:100), CHOP $(1: 1,000)$, IL-1 $\beta(1: 2,000)$, caspase-1 $(1: 1,000)$, caspase-11 (1:200), IL-18 (1:1,000), NLRP3 $(1: 1,000)$ and $\beta$-actin $(1: 200)$. The next morning, the PVDF membranes were washed three times with TBST and then incubated with secondary antibodies (horseradish peroxidase-conjugated goat anti-mouse and goat anti-rabbit; 1:50,000) for $2 \mathrm{~h}$ at $37^{\circ} \mathrm{C}$. Specific bands were detected by $\mathrm{ECL}^{\mathrm{TM}}$ (Beijing Pierce Biotechnology) and band densities were quantified using ImageJ software (v1.8.0; National Institutes of Health).

Statistical analysis. All data are expressed as the mean \pm SEM. Statistical analyses involved one-way ANOVA and Tukey's multiple comparisons tests. $\mathrm{P}<0.05$ was considered to indicate a statistically significant difference.

\section{Results}

Body weight and fasting blood glucose levels of normal and diabetic rats prior to renal IR injury. Five weeks after establishing the diabetic model, the diabetic rats exhibited characteristic symptoms of hyperglycemia, including polydipsia, polyphagia, polyuria and weight loss, compared with non-diabetic rats (Fig. 1). The blood glucose level of diabetic rats was significantly higher than that in non-diabetic rats, and the body weight of diabetic rats was significantly reduced $(\mathrm{P}<0.05)$.

Analyses of histopathology and renal function indicates that DM significantly aggravates renal $I / R$ injury. The rats in each group underwent either a sham operation or experienced ischemia for 45 min followed by reperfusion for $24 \mathrm{~h}$. As revealed in Fig. 2A and B, I/R injury significantly increased the tubular injury score which was used to evaluate the degree of kidney injury in both NI/R and DI/R groups $(\mathrm{P}<0.05$, compared with the NS group). Notably, renal pathological changes in the NI/R, DS, and DI/R groups exhibited significant damage in the renal tubules, as evidenced by the loss of brush border, swelling in the tubular epithelial cells and expansion of the interstitium $(\mathrm{P}<0.05)$. Most importantly, the DI/R group exhibited significantly more aggravated tissue damage than the NI/R group $(\mathrm{P}<0.05)$. As revealed in Fig. $2 \mathrm{C}$ and $\mathrm{D}, \mathrm{I} / \mathrm{R}$ injury-induced renal dysfunction led to a significant increase in the levels of Scr and BUN $(\mathrm{P}<0.05)$; these are parameters that are normally used to reflect renal function. Significantly higher levels of BUN and serum creatinine were observed in the $\mathrm{DI} / \mathrm{R}$ group than the NI/R group $(\mathrm{P}<0.05)$, demonstrating that the damage caused by IR was more severe in the DI/R group. These results illustrated that DM further aggravated I/R-induced damage in the kidney.
DM exacerbates renal I/R injury by enhancing ERS. The ERS response in diabetic and non-diabetic rats when subjected to renal I/R injury was next investigated by assessing PERK/eIF2 $\alpha /$ ATF4-mediated renal ERS. As revealed in Fig. 3A-E, the expression levels of p-PERK, p-eIF2 $\alpha$, ATF4, and CHOP were significantly higher in the diabetic kidney compared with the NS group $(\mathrm{P}<0.05)$. The DI/R group exhibited further exacerbation in terms of the ERS response compared with the NI/R group $(\mathrm{P}<0.05)$. These data indicated that an enhancement in ERS may be involved in the aggravation of renal I/R injury in DM rats.

Renal SIRT1 signaling is impaired in DM and pyroptosis is a crucial event during renal IR injury. The expression levels of SIRT1 and pyroptosis-related proteins were assessed in all experimental and control rats. As revealed in Fig. 4A and B, SIRT1 expression was significantly downregulated in the kidney following ischemia reperfusion injury in the DI/R group compared with the NS group $(\mathrm{P}<0.05)$. Furthermore, the expression levels of SIRT1 were significantly lower in the DI/R group compared with the NI/R group $(\mathrm{P}<0.05)$. As shown in Fig. 4A and C-H), pyroptosis-associated proteins, including caspase-1, caspase-11, NLRP3, IL-18, and IL-1 $\beta$, were markedly increased by I/R injury in the NI/R and DI/R groups compared with the NS group $(\mathrm{P}<0.05)$. In addition, following renal $\mathrm{I} / \mathrm{R}$, the levels of proteins related to pyroptosis were significantly higher in the diabetic group than those in the NI/R group $(\mathrm{P}<0.05)$. These data demonstrated that $\mathrm{DM}$ impaired renal SIRT1 signaling. Following renal I/R, the levels of SIRT1 were further reduced, thus triggering pyroptosis and resulting in $\mathrm{AKI}$.

Analysis of histopathology and renal function reveals that resveratrol, an established agonist of SIRT1, protects against renal I/R injury in diabetic rats. In order to further evaluate the effect of SIRT1 signaling on renal I/R injury in the animal model of type $1 \mathrm{DM}$, rats were pretreated with resveratrol, an established agonist of SIRT1, for 7 consecutive days prior to surgery. As revealed in Fig. 5A and B, I/R significantly increased the renal tubular injury scores related to pathological changes in the DI/R group compared with the DS group $(\mathrm{P}<0.05)$, while resveratrol pre-treatment effectively ameliorated renal injury in the DI/R+Res group compared with the DI/R group $(\mathrm{P}<0.05)$. As revealed in Fig. $5 \mathrm{C}$ and $\mathrm{D}$, significantly aggravated kidney dysfunction was evident in the $\mathrm{DI} / \mathrm{R}$ group compared with the DS group $(\mathrm{P}<0.05)$. However, resveratrol significantly reduced the levels of BUN and serum creatinine in the DI/R+Res group compared with the DI/R group $(\mathrm{P}<0.05)$. Collectively, these data indicated that the re-activation of SIRT1 partially protected against renal I/R injury in diabetic rats.

Resveratrol supplementation markedly increases SIRTI expression thus reducing the levels of ERS and alleviating renal pyroptosis. Next, the relationship between SIRT1 expression and ERS-mediated pyroptosis was explored in diabetic animals subjected to I/R injury. As revealed in Fig. $6 \mathrm{~F}$ and $\mathrm{G}$, $\mathrm{I} / \mathrm{R}$ injury resulted in an evident reduction in the expression of SIRT1 compared with the DS group $(\mathrm{P}<0.05)$. Moreover, resveratrol treatment led to a significantly higher level of SIRT1 
A

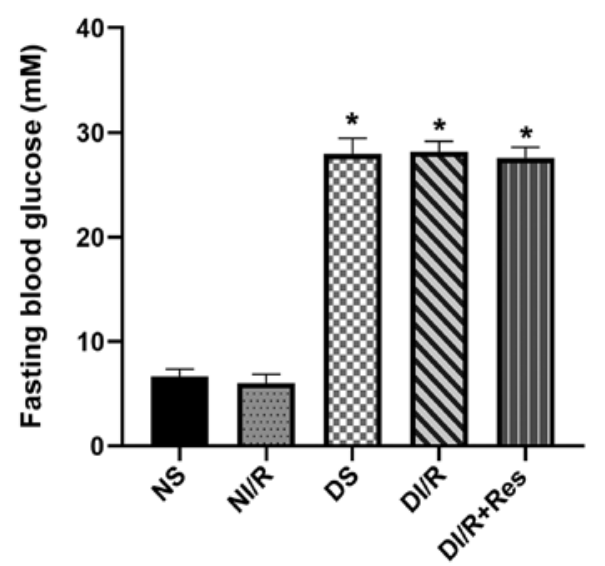

B

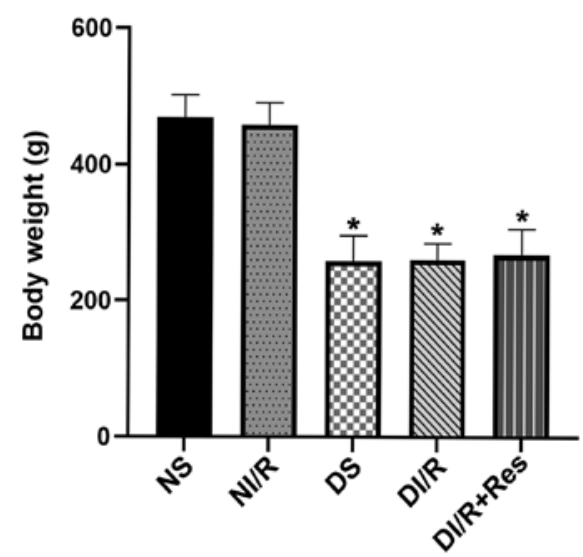

Figure 1. Comparison of fasting blood glucose levels and body weights between diabetic and non-diabetic rats. Five weeks after establishing the diabetic model, (A) fasting blood glucose levels and (B) body weights of diabetic and non-diabetic rats were assessed. The results are expressed as the mean \pm SEM, $\mathrm{n}=6$. ${ }^{*} \mathrm{P}<0.05$ vs. NS group and NI/R group. NS and DS groups received a sham operation. The left renal pedicles of rats in the NI/R and DI/R groups were clamped for $45 \mathrm{~min}$ with non-invasive vascular forceps followed by $24 \mathrm{~h}$ of unclamping for reperfusion. Rats in the DI/R+Res group received daily i.p. injections of resveratrol (10 mg/kg of body weight dissolved in DMSO and delivered with saline and 30\% ethanol.) for one week followed by another injection (i.p.) of the same dose $30 \mathrm{~min}$ before surgery. The groups assessed were as follows: NS, the non-diabetic sham group; NI/R, the non-diabetic I/R group; DS, the diabetic sham group; DI/R, the diabetic I/R group; DI/R+Res, the diabetic I/R + resveratrol group. I/R, ischemia/reperfusion; i.p., intraperitoneal; SEM, standard error of the mean.

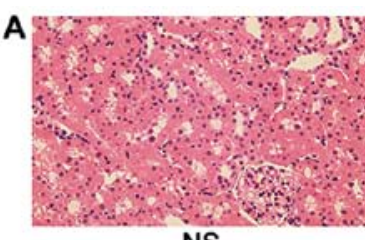

NS

B

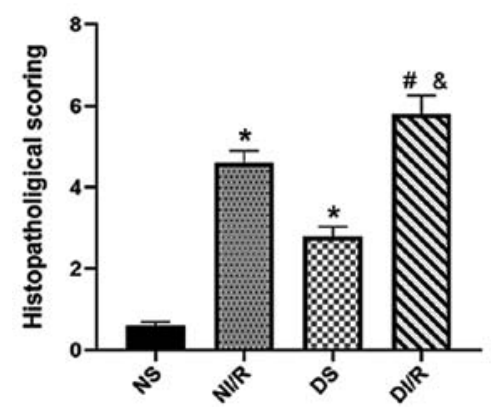

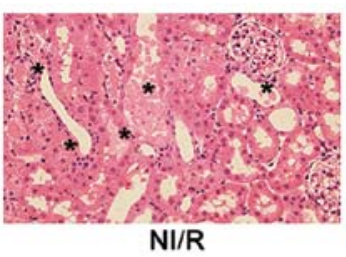

C

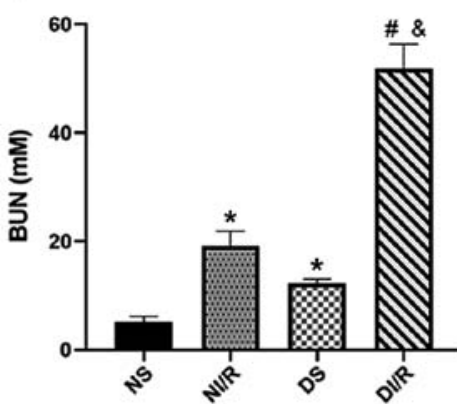

DS

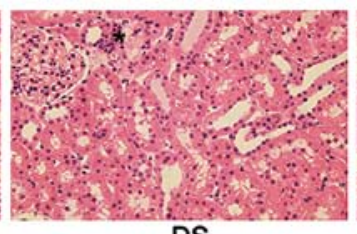

D

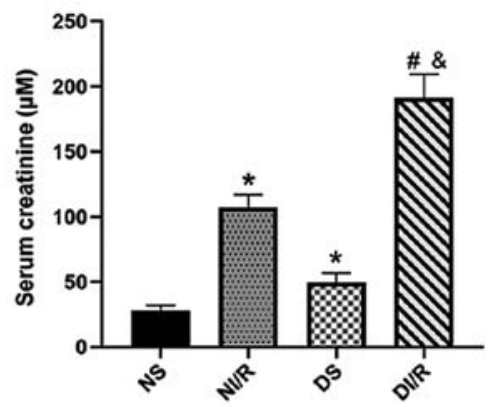

Figure 2. Rats were subjected to sham surgery or renal I/R injury. Twenty-four hours after reperfusion, serum and kidneys were collected for urea and creatinine testing and H\&E staining. (A) H\&E staining of kidney sections (magnification, $\mathrm{x} 400$ ); '*' symbol in the images of this part represents the pathological changes in the kidney, including tubular epithelial cell swelling, interstitial expansion, intertubular hemorrhaging and necrotic tubules. (B) Histopathological scoring. (C) Serum BUN concentration. (D) Serum creatinine concentration. Data are presented as the mean $\pm \mathrm{SEM}, \mathrm{n}=6 .{ }^{*} \mathrm{P}<0.05$ vs. NS group; ${ }^{*} \mathrm{P}<0.05$ vs. DS group; ${ }^{\&} \mathrm{P}<0.05$ vs. NI/R group. The groups assessed were as follows: NS, the non-diabetic sham group; NI/R, the non-diabetic I/R group; DS, the diabetic sham group; DI/R, the diabetic I/R group. I/R, ischemia/reperfusion; H\&E, hematoxylin and eosin; BUN, blood urea nitrogen; SEM, standard error of the mean.

in the DI/R+Res group than those in the DI/R group $(\mathrm{P}<0.05)$. As revealed in Fig. 6A-E, the activation of SIRT1 significantly reduced the expression of p-PERK, p-eIF2 $\alpha, \mathrm{ATF} 4$, and $\mathrm{CHOP}$ in the group treated with resveratrol $(\mathrm{P}<0.05)$. Furthermore, as revealed in Fig. 6F and H-M, the expression of caspase-1, caspase-11, IL-1 $\beta$, NLRP3, and IL-18 (which reflected the level of pyroptosis) was significantly reduced in the DI/R+Res group compared with the DI/R group $(\mathrm{P}<0.05)$. Collectively, these data further indicated that resveratrol suppressed renal ERS levels by upregulating SIRT1 signaling in diabetic rats.
Furthermore, the enhancement of SIRT1 resulted in the alleviation of renal pyroptosis.

\section{Discussion}

The present analysis indicated that SIRT1 signaling was impaired in type 1 diabetic rats. Following renal I/R injury, the levels of SIRT1 expression were further attenuated. Notably, resveratrol, a known agonist of SIRT1 signaling, alleviated ERS-mediated pyroptosis, resulting in the 
A
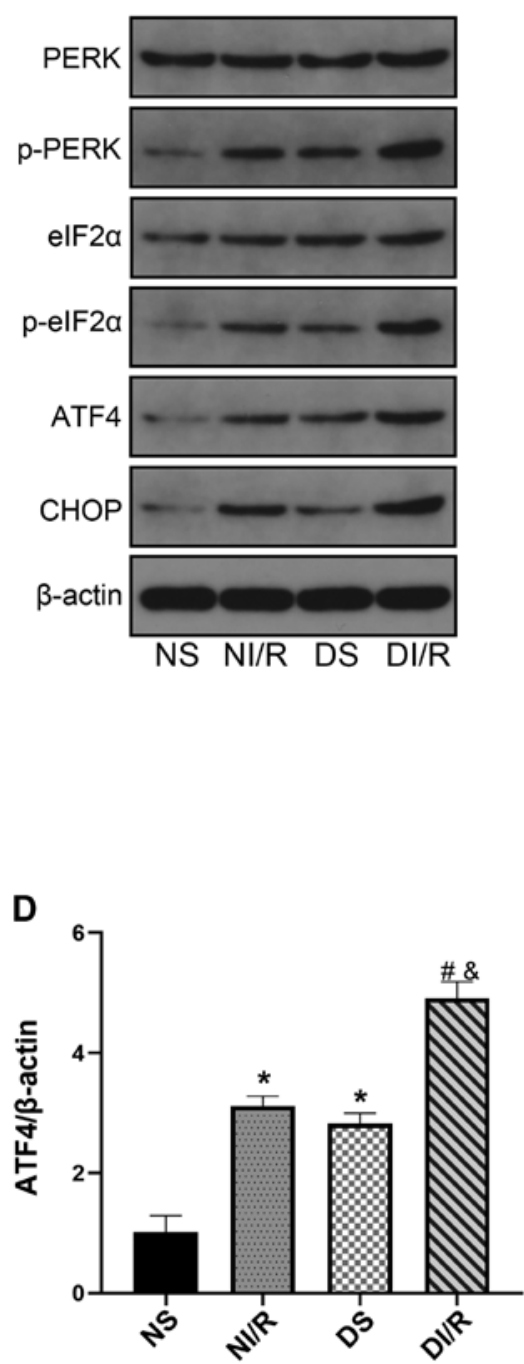

B

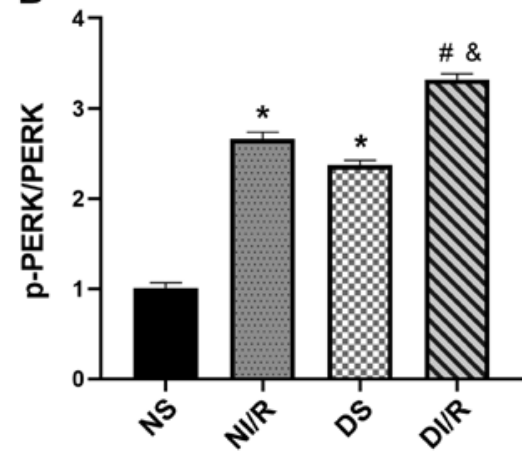

C

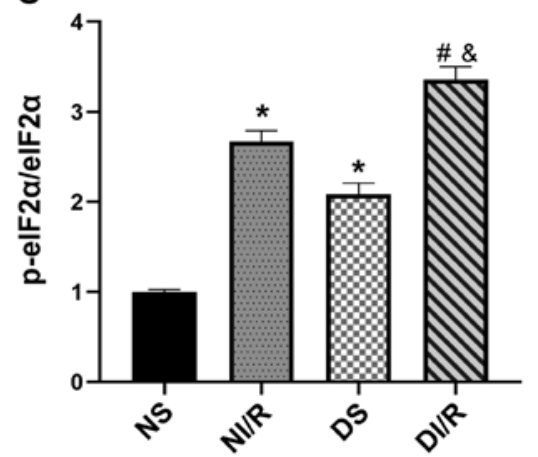

E

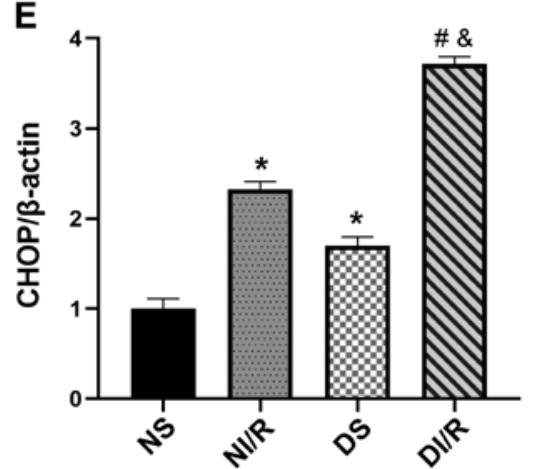

Figure 3. DM enhances endoplasmic reticulum stress. Western blot analysis was performed after $24 \mathrm{~h}$ of reperfusion. (A) Representative blots and histograms showing (B) p-PERK/PERK ratio; (C) p-eIF2 $\alpha /$ eIF2 $\alpha$ ratio; (D) ATF4 expression; and (E) CHOP expression. The results are expressed as the mean \pm SEM, $\mathrm{n}=6$. ${ }^{\prime} \mathrm{P}<0.05$ vs. NS group; ${ }^{\prime} \mathrm{P}<0.05$ vs. DS group; ${ }^{\&} \mathrm{P}<0.05$ vs. NI/R group. The groups assessed were as follows: NS, the non-diabetic sham group; NI/R, the non-diabetic I/R group; DS, the diabetic sham group; DI/R, the diabetic I/R group. DM, diabetes mellitus; SEM, standard error of the mean; I/R, ischemia/reperfusion; $\mathrm{p}$-, phosphorylated; eIF2 $\alpha$, eukaryotic translation initiation factor 2 subunit $\alpha$; ATF4, activating transcription factor 4; CHOP, C/EBP homologous protein.

amelioration of I/R-induced kidney injury. Fig. 7 outlines the proposed induction of pyroptosis. In the diagram renal injury in patients with DM induces hyperglycemia, while also initiating ROS and an inflammatory response, as well as downregulating the expression of SIRT1 (a protein that normally protects the ER from stress). This results in ER stress via the PERK/eIF2 $\alpha /$ ATF4/CHOP pathways, thus triggering pyroptosis. The present data therefore revealed a potential mechanism for the exacerbation of renal injury following $I / R$ in diabetics.

Renal I/R injury can induce dysfunction in a range of organs, including AKI (30). Furthermore, clinical trials have demonstrated that AKI is associated with high morbidity rates (31). It is well known that DM is a serious risk factor for renal disease (32) and that renal I/R injury, accompanied by diabetes, can aggravate AKI; this condition has a poor prognosis (33). Previous studies have reported that the accumulation of ROS plays a significant role in I/R-induced kidney injury in diabetic patients (34). A previous study reported increased levels of BUN, serum creatinine and proinflammatory cytokines in diabetic rats that had experienced $I / R$ injury (6). In the present study, it was observed that diabetes aggravated renal I/R injury through acute tubular damage and exacerbated kidney dysfunction; these effects were reflected by the higher levels of BUN and serum creatinine. These results were in line with those reported by previous publications.

SIRT1, an $\mathrm{NAD}^{+}$-dependent deacetylase, can exert a marked effect in a number of cellular functions, including transcriptional reprogramming, DNA repair, stress resistance and apoptosis (35). In a recent study, Li et al (21) demonstrated that SIRT1 is a significant age-related protective factor against renal I/R-induced injury. Moreover, several studies have 
A

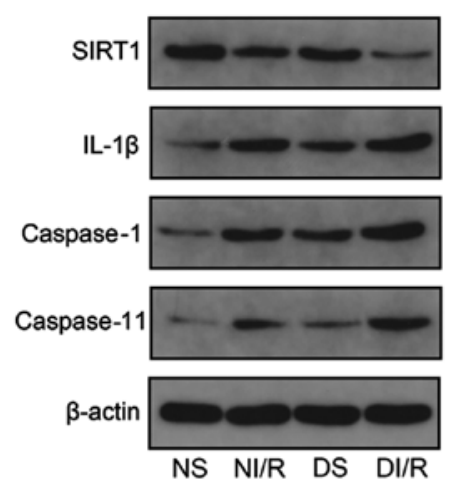

$\mathbf{F}$

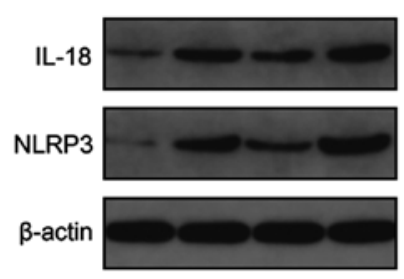

B
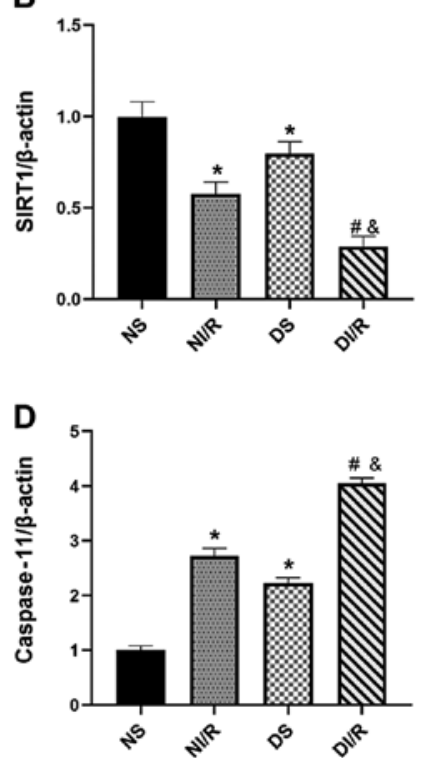

G

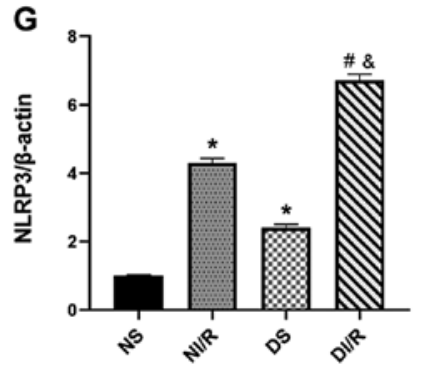

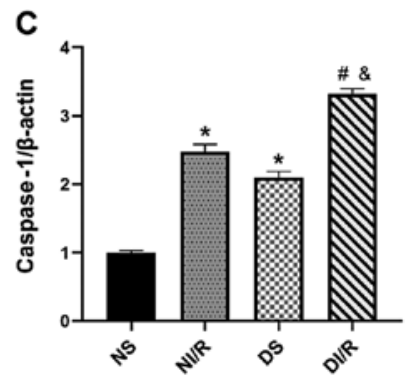
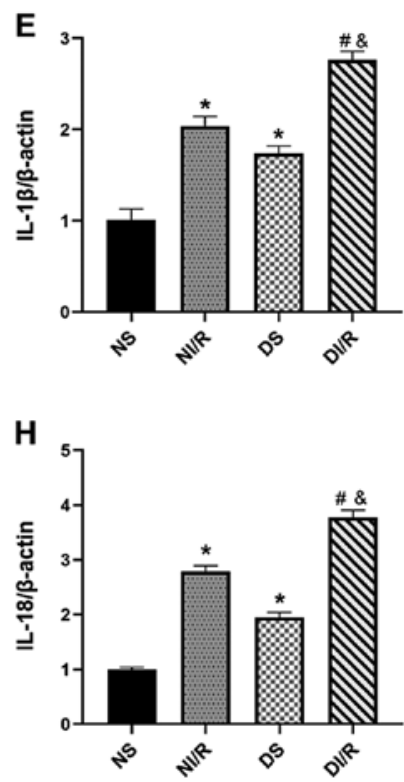

Figure 4. DM impairs myocardial SIRT1 signaling and aggravates pyroptosis in both renal I/R-injured and control rats. Western blot analysis was performed after $24 \mathrm{~h}$ of reperfusion. (A) Representative blots and histograms showing expression of (B) SIRT1; (C) active caspase-1; (D) active caspase-11; and (E) IL-1 3 . (F) Representative blots. Histograms showing expression of (G) NLRP3 and (H) IL-18. The results are expressed as the mean \pm SEM, $n=6$. "P<0.05 vs. NS group; ${ }^{*} \mathrm{P}<0.05$ vs. DS group; ${ }^{*} \mathrm{P}<0.05$ vs. NI/R group. The groups assessed were as follows: NS, the non-diabetic sham group; NI/R, the non-diabetic I/R group; DS, the diabetic sham group; DI/R, the diabetic I/R group. DM, diabetes mellitus; SIRT1, sirtuin 1; I/R, ischemia/reperfusion; SEM, standard error of the mean; IL, interleukin; NLRP3, NLR family pyrin domain containing 3.

A

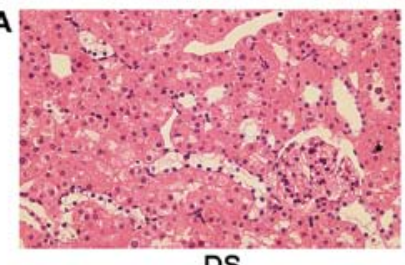

DS

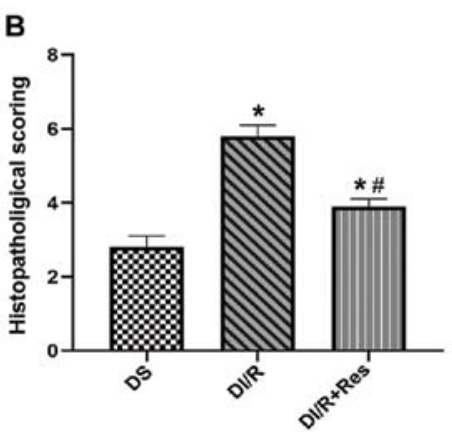

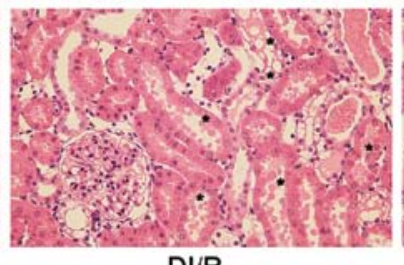

$\mathrm{DI} / \mathrm{R}$

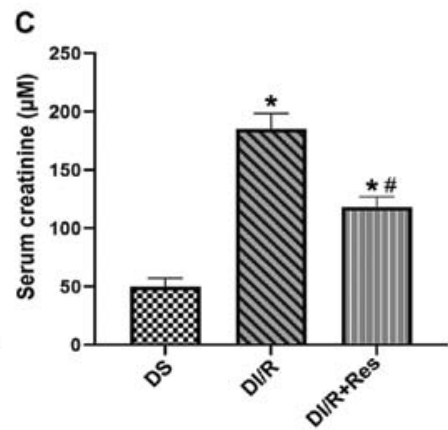

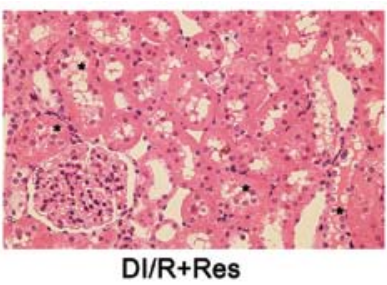

D

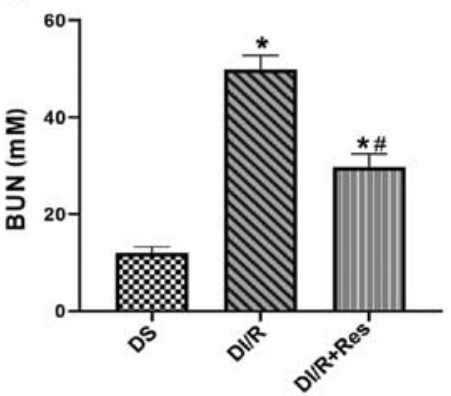

Figure 5. Resveratrol pre-treatment significantly attenuates kidney dysfunction in terms of the serum levels of creatine, BUN and histopathological scoring. (A) H\&E staining of kidney sections (magnification, $\mathrm{x} 400$ ); ' $*$ ' symbol represents the pathological changes in the kidney, including tubular epithelial cell swelling, interstitial expansion, intertubular hemorrhaging and necrotic tubules. (B) Histopathological scoring. (C) Serum creatinine concentration. (D) Serum BUN concentration. Data are presented as the mean \pm SEM. ${ }^{*} \mathrm{P}<0.05$ vs. the $\mathrm{DS}$ group; ${ }^{~} \mathrm{P}<0.05$ vs. the DI/R group. The groups assessed were as follows: $\mathrm{DS}$, the diabetic sham group; DI/R, the diabetic I/R group; DI/R+Res, the diabetic I/R + resveratrol group. BUN, blood urea nitrogen; I/R, ischemia/reperfusion; SEM, standard error of the mean. 
A

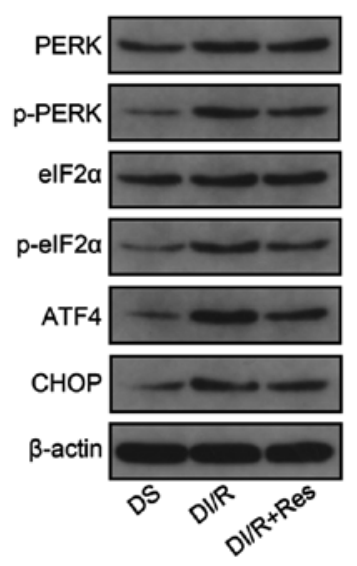

$\mathbf{F}$
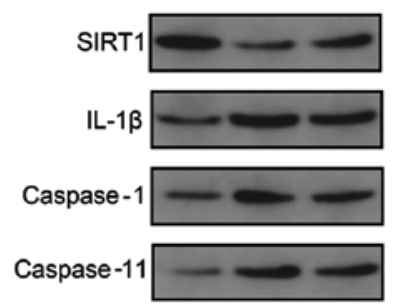

$\beta$-actin

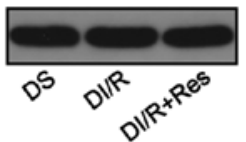

K

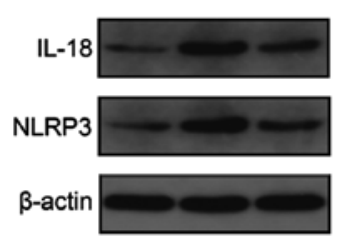

B

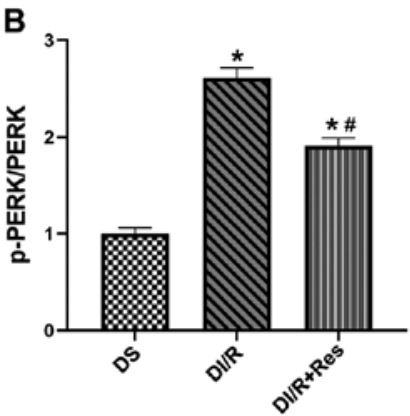

D

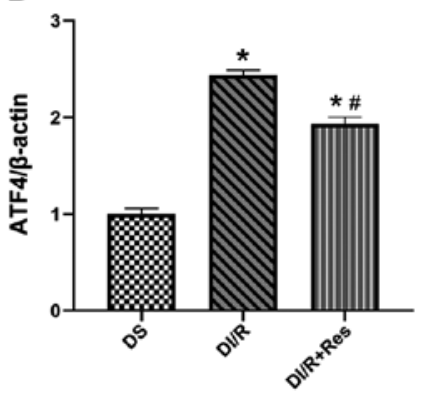

G
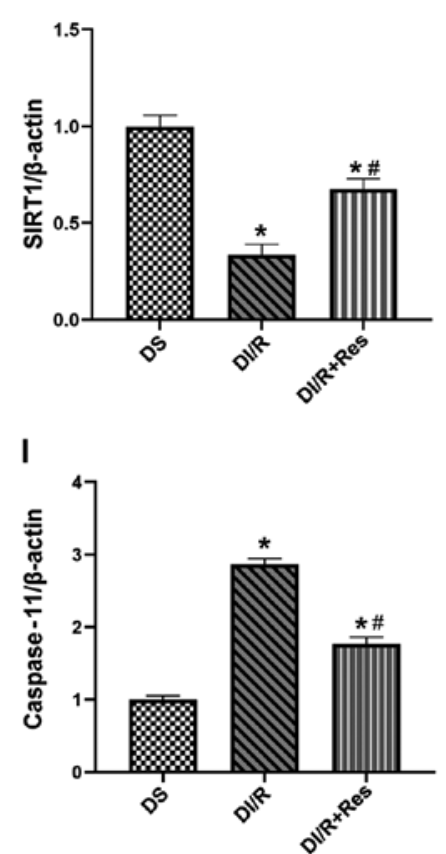

L

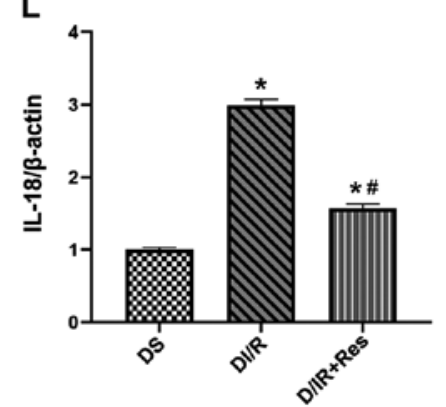

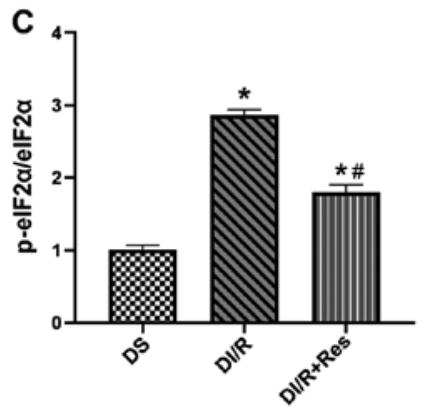

E

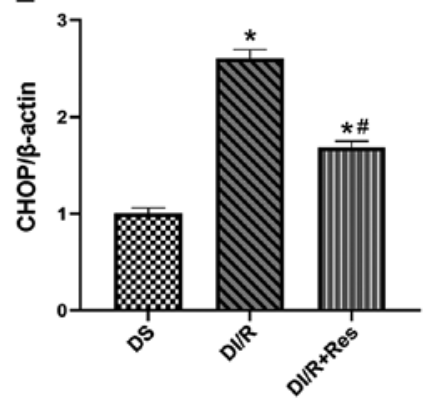

H

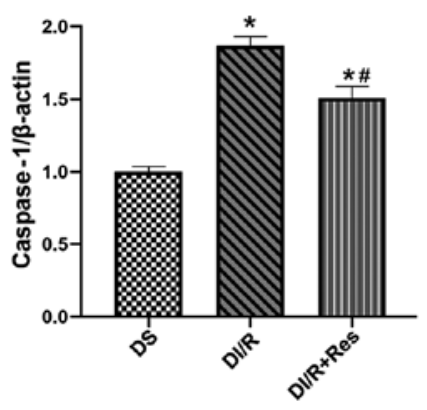

J

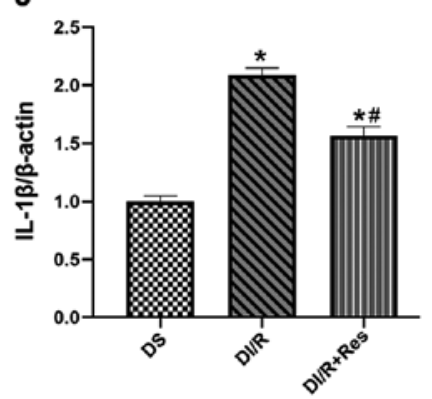

M

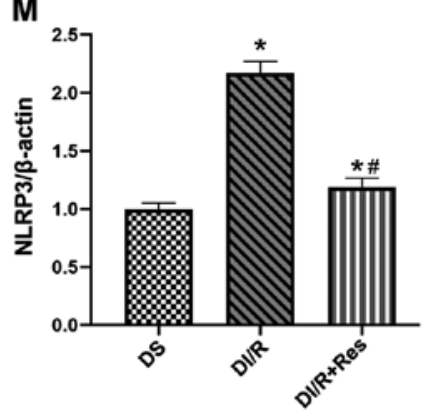

Figure 6. Resveratrol pre-treatment upregulates SIRT1 expression, reduces endoplasmic reticulum stress and attenuates pyroptosis induced by I/R injury in diabetic rats. Western blotting was performed, (A) representative blots and histograms showing (B) p-PERK/PERK ratio; (C) p-eIF2 $\alpha /$ IF2 $\alpha$ ratio; (D) ATF4 expression; and (E) CHOP expression. (F) Representative blots and histograms showing expression of (G) SIRT1; (H) active caspase-1; (I) active caspase-11; and (J) IL-1 1 . (K) Representative blots and histograms showing expression of (L) IL-18 and (M) NLRP3. Date are expressed as the mean \pm SEM, $n=6 .{ }^{*} \mathrm{P}<0.05$ vs. DS group; ${ }^{*} \mathrm{P}<0.05$ vs. DI/R group. The groups assessed were as follows: $\mathrm{DS}$, the diabetic sham group; DI/R, the diabetic I/R group; DI/R+Res, the diabetic I/R + resveratrol group. SIRT1, sirtuin 1; I/R, ischemia/reperfusion; SEM, standard error of the mean; p, phosphorylated; eIF2 $\alpha$, eukaryotic translation initiation factor 2 subunit $\alpha$; ATF4, activating transcription factor 4; CHOP, C/EBP homologous protein; IL, interleukin; NLRP3, NLR family pyrin domain containing 3 . 


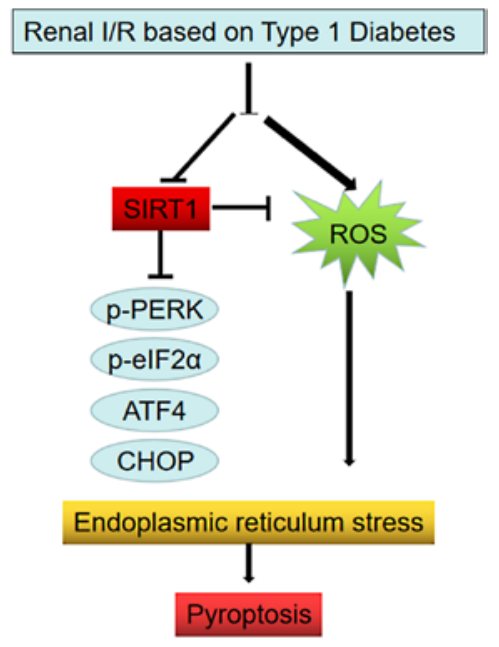

Figure 7. Schematic diagram of the induction of pyroptosis via ER stress and the effects of SIRT1 on this process. DM induces hyperglycemia and initiates cellular oxidative stress and an inflammatory response. Collectively, these processes result in ER stress via the PERK/eIF2 $\alpha / \mathrm{ATF} 4 / \mathrm{CHOP}$ pathways. Furthermore, DM downregulated the expression of SIRT1, a protein that normally protects the ER from stress. ER, endoplasmic reticulum; SIRT1, sirtuin 1; DM, diabetes mellitus; I/R, ischemia/reperfusion; $p$, phosphorylated; eIF $2 \alpha$, eukaryotic translation initiation factor 2 subunit $\alpha$ ATF4, activating transcription factor 4; CHOP, C/EBP homologous protein; ROS, reactive oxygen species.

demonstrated that SIRT1 may represent an effective therapeutic option for diabetes by controlling insulin secretion, regulating fatty acid oxidation $(36,37)$ and defending against cellular oxidative damage and inflammation (38). Notably, Yu et al (39) demonstrated that DM downregulated SIRT1 signaling, and it has been shown that this effect was further impaired by I/R injury in cardiomyocytes (39). In accordance with previous research, in the present study it was observed that the expression of SIRT1 was decreased in both I/R groups, especially in the DI/R group. These results indicated that diabetes leads to a reduction in SIRT1 signaling, thus exacerbating the damage caused by renal I/R injury-induced oxidative stress. It was revealed that IR injury-induced kidney dysfunction and tissue damage in diabetic rats was markedly alleviated by treatment with resveratrol, a known agonist of SIRT1. Collectively, this data supported the hypothesis that SIRT1 was notably attenuated in diabetic rats and was further impaired by $I / R$ injury. However, the reason that I/R treatment downregulates the expression of SIRT1 may be due to the overactivation of oxidative stress or other factors, and the underlying mechanism requires further exploration in a future study.

Numerous research studies have verified the relevance of ERS in the pathophysiological process of diabetic I/R injury in cardiomyocytes $(40,41)$. For example, Yu et al (39) revealed that the inhibition of oxidative stress, and the attenuation of SIRT1-mediated ERS, could improve myocardial I/R-induced damage in diabetic state. More recently, Guo et al (42) revealed that ERS levels were downregulated by SIRT1 in diabetic rats. However, very little is known about kidney injury. In the present study, it was observed that the upregulation of ERS was mediated by the PERK/eIF2 $\alpha /$ ATF4 pathway in both of the I/R groups but especially in the DI/R group. In addition, resveratrol was used in the DI/R groups to further confirm the effects of SIRT1. As anticipated, the SIRT1 agonist attenuated the ERS levels. To the best of our knowledge, there is no previous research describing the fact that DM exacerbates renal I/R injury by enhancing SIRT1-mediated levels of ERS. In addition, the specific mechanisms responsible for the effect of SIRT1 on ERS in cases of I/R injury in the diabetic kidney have yet to be fully elucidated. Previous studies highlighted the potential role of SIRT1, predominantly because this protein is associated with the circulatory system and has the ability to regulate antioxidative stress $(43,44)$. It was speculated in the present study that DM impaired SIRT1 signaling and that the increased levels of oxidative stress in diabetic rats may contribute to enhanced ERS via the PERK/eIF2 $\alpha /$ ATF4 pathway, thus leading to the aggravation of $I / R$ injury-induced kidney damage.

A previous study has demonstrated that via the activation ERS, I/R injury can induce several types of cell death, including autophagy, apoptosis, and necroptosis (20). Pyroptosis, which is dependent upon the levels of caspase-1, can cause the plasma membrane to burst and the activation of a range of inflammatory mediators; this results in a form of inflammatory cell death that differs from apoptosis $(20,45)$. Furthermore, the activation of caspase- 1 can result in the separate conversion of pro-inflammatory forms of IL-1 $\beta$ and IL-18 into mature IL-1 $\beta$ and IL-18 (46). Subsequently, these active inflammatory cytokines are delivered to the internal environment to promote inflammation (47). Qiu et al (48) further demonstrated that the activation of inflammatory mediators, including caspase-1, IL-1 $\beta$ and IL-18 was elevated in a diabetic animal model. Furthermore, this study showed that when these diabetic rats were subjected to myocardial I/R insult, there were further increases in the levels of NLRP3 inflammasomes, activated caspase-1 and IL-1 $\beta$. In another study, Wang et al (49) revealed that pyroptosis was associated with the development of $\mathrm{I} / \mathrm{R}$ in renal tubular cells. The present study revealed that diabetes and ischemia both significantly induce cellular pyroptosis and that this effect was exacerbated in diabetic states. It was also revealed that resveratrol ameliorated pyroptosis-mediated renal damage. Previous studies reported that ERS is an essential pathway in pyroptosis $(50,20)$. The present data concurred with these previous findings in that the increased expression of caspase-1, caspase- 11 and IL- $1 \beta$ was observed in the NI/R group, especially in the DI/R group. In addition, resveratrol ameliorated this effect. Collectively, the data generated during this study indicated that DM aggravates renal I/R injury by downregulating the SIRT1 pathway. However, diabetes is a complex metabolic disease involving aberrant levels of glucose, lipids and inflammation (3,7). As for the specific factors of diabetes that may be associated with the SIRT1 pathway and ERS, further research is required.

The experimental data of the present study revealed that SIRT1 signaling was impaired in diabetic rats, thus aggravating ERS. This induced cellular pyroptosis following renal I/R injury, which ultimately led to AKI. The present research enhanced our understanding of why the diabetic kidney is susceptible to I/R injury. In addition, SIRT1 appears to represent a promising therapeutic target for diabetic patients with renal I/R injury.

\section{Acknowledgements}

Not applicable. 


\section{Funding}

The present study was supported by The Wuhan Morning Light Plan of Youth Science and Technology (grant no. 2017050304010281), The Natural Science Foundation of Hubei Province (grant nos. 2016CFB114 and 2017CFB181) and The Research Project of Wuhan University (grant no. 2042017kf0097).

\section{Availability of data and materials}

The datasets used and/or analyzed during the current study are available from the corresponding author on reasonable request.

\section{Authors' contributions}

JZ, DG, YY and LW conceived and designed the research, performed experiments and approved the final version of the manuscript. JZ, ZC and XL interpreted the results and prepared the figures. JZ, XL and LW analyzed the data, drafted, edited and revised the manuscript. All authors reviewed and approved the final manuscript, certify that they have participated sufficiently in the present study, and agree to be accountable for all aspects of the research in ensuring that the accuracy or integrity of any part of the work are appropriately investigated and resolved.

\section{Ethics approval and consent to participate}

This study was approved by The Ethics Committee of Renmin Hospital of Wuhan University, and all procedures complied with the Principles of Animal Care of Wuhan University (Wuhan, China) and Guide for the Care and Use of Laboratory Animals.

\section{Patient consent for publication}

Not applicable.

\section{Competing interests}

The authors declare that they have no competing interests.

\section{References}

1. Li PK, Burdmann EA and Mehta RL: World kidney day 2013 Acute kidney injury-global health alert. Am J Kidney Dis 61 359-363, 2013.

2. Wang X, Bonventre JV and Parrish AR: The aging kidney: Increased susceptibility to nephrotoxicity. Int J Mol Sci 15 15358-15376, 2014.

3. Lejay A, Fang F, John R, Van JA, Barr M, Thaveau F, Chakfe N, Geny B and Scholey JW: Ischemia reperfusion injury, ischemic conditioning and diabetes mellitus. J Mol Cell Cardiol 91: 11-22, 2016.

4. Muroya Y, He X, Fan L, Wang S, Xu R, Fan F and Roman RJ: Enhanced renal ischemia-reperfusion injury in aging and diabetes. Am J Physiol Renal Physiol 315: F1843-F1854, 2018.

5. Melin J, Hellberg O, Akyurek LM, Kallskog O, Larsson E and Fellstrom BC: Ischemia causes rapidly progressive nephropathy in the diabetic rat. Kidney Int 52: 985-991, 1997.

6. Zhang Y, Hu F, Wen J, Wei X, Zeng Y, Sun Y, Luo S and Sun L: Effects of sevoflurane on NF- $\mathrm{BB}$ and TNF- $\alpha$ expression in renal ischemia-reperfusion diabetic rats. Inflamm Res 66: 901-910, 2017.
7. Shi S, Lei S, Tang C, Wang K and Xia Z: Melatonin attenuates acute kidney ischemia/reperfusion injury in diabetic rats by activation of the SIRT1/Nrf2/HO-1 signaling pathway. Biosci Rep 39: BSR20181614, 2019.

8. Qiu Y, Wu Y, Meng M, Luo M, Zhao H, Sun H and Gao S GYY4137 protects against myocardial ischemia/reperfusion injury via activation of the PHLPP-1/Akt/Nrf2 signaling pathway in diabetic mice. J Surg Res 225: 29-39, 2018.

9. Bravo R, Parra V, Gatica D, Rodriguez AE, Torrealba N, Paredes F, Wang ZV, Zorzano A, Hill JA, Jaimovich E, et al: Endoplasmic reticulum and the unfolded protein response: Dynamics and metabolic integration. Int Rev Cell Mol Biol 301: 215-290, 2013.

10. Gao X, Fu L, Xiao M, Xu C, Sun L, Zhang T, Zheng F and Mei C: The nephroprotective effect of tauroursodeoxycholic acid on ischaemia/reperfusion-induced acute kidney injury by inhibiting endoplasmic reticulum stress. Basic Clin Pharmacol Toxicol 111: 14-23, 2012.

11. Gu Y, Huang F, Wang Y, Chen C, Wu S, Zhou S, Hei Z and Yuan D: Connexin 32 plays a crucial role in ROS-mediated endoplasmic reticulum stress apoptosis signaling pathway in ischemia reperfusion-induced acute kidney injury. J Transl Med 16: 117, 2018.

12. Yan M, Shu S, Guo C, Tang C and Dong Z: Endoplasmic reticulum stress in ischemic and nephrotoxic acute kidney injury. Ann Med 50: 381-390, 2018.

13. Liu H, Wang L, Weng X, Chen H, Du Y, Diao C, Chen Z and Liu X: Inhibition of Brd4 alleviates renal ischemia/reperfusion injury-induced apoptosis and endoplasmic reticulum stress by blocking FoxO4-mediated oxidative stress. Redox Biol 24: 101195, 2019.

14. Su M, Ren S, Zhong W and Han X: Impact of propofol on renal ischemia/reperfusion endoplasmic reticulum stress. Acta Cir Bras 32: 533-539, 2017.

15. Zhu H, Fan Y, Sun H, Chen L and Man X: Curcumin inhibits endoplasmic reticulum stress induced by cerebral ischemiareperfusion injury in rats. Exp Ther Med 14: 4047-4052, 2017.

16. Pandey VK, Mathur A and Kakkar P: Emerging role of Unfolded Protein Response (UPR) mediated proteotoxic apoptosis in diabetes. Life Sci 216: 246-258, 2019.

17. Man SM, Karki R and Kanneganti TD: Molecular mechanisms and functions of pyroptosis, inflammatory caspases and inflammasomes in infectious diseases. Immunol Rev 277: 61-75, 2017.

18. Dong T, Liao D, Liu X and Lei X: Using small molecules to dissect non-apoptotic programmed cell death: Necroptosis, ferroptosis, and pyroptosis. Chembiochem 16: 2557-2561, 2015.

19. Jorgensen I, Lopez JP, Laufer SA and Miao EA: IL-1 $\beta$, IL-18, and eicosanoids promote neutrophil recruitment to pore-induced intracellular traps following pyroptosis. Eur J Immunol 46: 2761-2766, 2016.

20. Yang JR, Yao FH, Zhang JG, Ji ZY, Li KL, Zhan J, Tong YN, Lin LR and He YN: Ischemia-reperfusion induces renal tubule pyroptosis via the CHOP-caspase-11 pathway. Am J Physiol Renal Physiol 306: F75-F84, 2014.

21. Li WF, Yang K, Zhu P, Zhao HQ, Song YH, Liu KC and Huang WF: Genistein ameliorates ischemia/reperfusion-induced renal injury in a SIRT1-dependent manner. Nutrients 9: E403, 2017.

22. Kelly GS: A review of the sirtuin system, its clinical implications, and the potential role of dietary activators like resveratrol: Part 2. Altern Med Rev 15: 313-328, 2010.

23. Prola A, Pires Da Silva J, Guilbert A, Lecru L, Piquereau J, Ribeiro M, Mateo P, Gressette M, Fortin D, Boursier C, et al: SIRT1 protects the heart from ER stress-induced cell death through eIF2 $\alpha$ deacetylation. Cell Death Differ 24: 343-356, 2017.

24. Koka S, Aluri HS, Xi L, Lesnefsky EJ and Kukreja RC: Chronic inhibition of phosphodiesterase 5 with tadalafil attenuates mitochondrial dysfunction in type 2 diabetic hearts: Potential role of NO/SIRT1/PGC-1 $\alpha$ signaling. Am J Physiol Heart Circ Physiol 306: H1558-H1568, 2014.

25. Yu L, Sun Y, Cheng L, Jin Z, Yang Y, Zhai M, Pei H, Wang X, Zhang H, Meng Q, et al: Melatonin receptor-mediated protection against myocardial ischemia/reperfusion injury: Role of SIRT1. J Pineal Res 57: 228-238, 2014.

26. Xue R, Lei S, Xia ZY, Wu Y, Meng Q, Zhan L, Su W, Liu H, Xu J, Liu Z, et al: Selective inhibition of PTEN preserves ischaemic post-conditioning cardioprotection in STZ-induced Type 1 diabetic rats: Role of the PI3K/Akt and JAK2/STAT3 pathways. Clin Sci (Lond) 130: 377-392, 2016. 
27. Zhang W, Luo J, Yang F, Wang Y, Yin Y, Strom A, Gustafsson JA and Guan X: BRCA1 inhibits AR-mediated proliferation of breast cancer cells through the activation of SIRT1. Sci Rep 6: 22034, 2016

28. Sun J, Guo E, Yang J, Yang Y, Liu S, Hu J, Jiang X, Dirsch O, Dahmen U, Dong W and Liu A: Carbon monoxide ameliorates hepatic ischemia/reperfusion injury via sirtuin 1-mediated deacetylation of high-mobility group box 1 in rats. Liver Transpl 23 : 510-526, 2017.

29. Xie Y,Jiang D, Xiao J,Fu C,Zhang Z, YeZ and Zhang X: Ischemic preconditioning attenuates ischemia/reperfusion-induced kidney injury by activating autophagy via the SGK1 signaling pathway. Cell Death Dis 9: 338, 2018.

30. Wang L, Liu X, Chen H, Chen Z, Weng X, Qiu T and Liu L: Effect of picroside II on apoptosis induced by renal ischemia/reperfusion injury in rats. Exp Ther Med 9: 817-822, 2015.

31. Wu MY, Yiang GT, Liao WT, Tsai AP, Cheng YL, Cheng PW, Li CY and Li CJ: Current mechanistic concepts in ischemia and reperfusion injury. Cell Physiol Biochem 46: 1650-1667, 2018.

32. Melin J, Hellberg $\mathrm{O}$ and Fellström B: Hyperglycaemia and renal ischaemia-reperfusion injury. Nephrol Dial Transplant 18: 460-462, 2003.

33. Kumas M, Essrefoğlu M, Karatas E, Duymaç N, Kanbay S, Ergün IS, Üyüklü M and Koçyiğit A: Investigation of dose-dependent effects of berberine against renal ischemia/reperfusion injury in experimental diabetic rats. Nefrologia 39: 411-423, 2019 (In Spanish).

34. Abu-Saleh N, Awad H, Khamaisi M, Armaly Z, Karram T, Heyman SN, Kaballa A, Ichimura T, Holman J and Abassi Z: Nephroprotective effects of TVP1022, a non-MAO inhibitor $\mathrm{S}$-isomer of rasagiline, in an experimental model of diabetic renal ischemic injury. Am J Physiol Renal Physiol 306: F24-F33, 2014.

35. Grubisha O, Smith BC and Denu JM: Small molecule regulation of Sir2 protein deacetylases. FEBS J 272: 4607-4616, 2005.

36. Gerhart-Hines Z, Rodgers JT, Bare O, Lerin C, Kim SH, Mostoslavsky R, Alt FW, Wu Z and Puigserver P: Metabolic control of muscle mitochondrial function and fatty acid oxidation through SIRT1/PGC-1alpha. EMBO J 26: 1913-1923, 2007.

37. Bordone L, Motta MC, Picard F, Robinson A, Jhala US, Apfeld J, McDonagh T, Lemieux M, McBurney M, Szilvasi A, et al: Sirt1 regulates insulin secretion by repressing $\mathrm{UCP} 2$ in pancreatic beta cells. PLoS Biol 4: e31, 2006.

38. Kitada M and Koya D: SIRT1 in Type 2 diabetes: Mechanisms and therapeutic potential. Diabetes Metab J 37: 315-325, 2013

39. Yu L, Liang H, Dong X, Zhao G, Jin Z, Zhai M, Yang Y, Chen W, Liu J, Yi W, et al: Reduced silent information regulator 1 signaling exacerbates myocardial ischemia-reperfusion injury in type 2 diabetic rats and the protective effect of melatonin J Pineal Res 59: 376-390, 2015.
40. Xu J, Zhou Q, Xu W and Cai L: Endoplasmic reticulum stress and diabetic cardiomyopathy. Exp Diabetes Res 2012: 827971, 2012.

41. Guo W, Jiang T, Lian C, Wang H, Zheng Q and Ma H: QKI deficiency promotes FoxO1 mediated nitrosative stress and endoplasmic reticulum stress contributing to increased vulnerability to ischemic injury in diabetic heart. J Mol Cell Cardiol 75: 131-140, 2014.

42. Guo R, Liu W, Liu B, Zhang B, Li W and Xu Y: SIRT1 suppresses cardiomyocyte apoptosis in diabetic cardiomyopathy: An insight into endoplasmic reticulum stress response mechanism. Int J Cardiol 191: 36-45, 2015.

43. Chong ZZ, Wang S, Shang YC and Maiese K: Targeting cardiovascular disease with novel SIRT1 pathways. Future Cardiol 8: 89-100, 2012.

44. Luo XY, Qu SL, Tang ZH, Zhang Y, Liu MH, Peng J, Tang H, Yu KL, Zhang C, Ren Z and Jiang ZS: SIRT1 in cardiovascular aging. Clin Chim Acta 437: 106-114, 2014.

45. Bergsbaken $\mathrm{T}$ and Cookson BT: Macrophage activation redirects yersinia-infected host cell death from apoptosis to caspase-1-dependent pyroptosis. PLoS Pathog 3: e161, 2007.

46. Fantuzzi G and Dinarello CA: Interleukin-18 and interleukin-1 beta: Two cytokine substrates for ICE (caspase-1). J Clin Immunol 19: 1-11, 1999.

47. Chou X, Ding F, Zhang X, Ding X, Gao H and Wu Q: Sirtuin-1 ameliorates cadmium-induced endoplasmic reticulum stress and pyroptosis through XBP-1s deacetylation in human renal tubular epithelial cells. Arch Toxicol 93: 965-986, 2019.

48. Qiu Z, Lei S, Zhao B, Wu Y, Su W, Liu M, Meng Q, Zhou B, Leng Y and Xia ZY: NLRP3 Inflammasome activation-mediated pyroptosis aggravates myocardial ischemia/reperfusion injury in diabetic rats. Oxid Med Cell Longev 2017: 9743280, 2017.

49. Wang L, Chen Z, Weng X, Wang M, Du Y and Liu X: Combined ischemic postconditioning and ozone postconditioning provides synergistic protection against renal ischemia and reperfusion injury through inhibiting pyroptosis. Urology 123: 296.e1-296.e8, 2019.

50. Yang CC, Yao CA, Yang JC and Chien CT: Sialic acid rescues repurified lipopolysaccharide-induced acute renal failure via inhibiting TLR4/PKC/gp91-mediated endoplasmic reticulum stress, apoptosis, autophagy, and pyroptosis signaling. Toxicol Sci 141: 155-165, 2014. 